\title{
EVALUATION OF CERTAIN INTEGRALS INVOLVING BESSEL FUNCTIONS
}

\author{
by E. DEUTSCH
}

(Received 3rd March 1963)

\section{Introduction}

In the solution of boundary value problems in mathematical physics by means of integral transforms we often find that the solution of a particular problem can be expressed in terms of integrals of the type

$$
G_{m, n}(r, z)=\int_{0}^{\infty} t^{m-2} e^{-t z}(\sin a t-a t \cos a t) J_{n}(r t) d t
$$

where $r$ and $z$ are positive and $m$ and $n$ are integers satisfying the convergence condition $m+n>-2$.

As examples of the use of these integrals in elasticity we may quote the following (1), (2):

(i) The state of stress in a semi-infinite elastic medium $z \geqq 0$ deformed by there being pressed against it a perfectly rigid axially symmetric punch of approximately spherical (paraboloidal) shape.

(ii) The torsional displacements in a semi-infinite elastic medium when the surface values are prescribed over a circular area at the boundary of the semiinfinite body, while the region of the surface lying outside this circle is free from stress.

(iii) The stress in an infinite elastic medium containing a penny-shaped crack, the crack being deformed by the action of a constant pressure.

On using the formula

$$
J_{\frac{3}{2}}(x)=\left(\frac{2}{\pi x}\right)^{\frac{1}{2}}\left(\frac{\sin x}{x}-\cos x\right),
$$

the integral (1.1) may be written

$$
G_{m, n}(r, z)=\left(\frac{\pi}{2}\right)^{\frac{1}{2}} a^{\frac{3}{2}} \int_{0}^{\infty} t^{m-\frac{1}{2}} e^{-t z} J_{\frac{z}{2}}(a t) J_{n}(r t) d t,
$$

whence it follows (3) that these integrals are convergent if $z>0$ and $m+n>-2$. If $z=0$ then we must have

$$
\begin{aligned}
& m+n>-2 \text { and } m<\frac{3}{2} \text { if } r \neq a \\
& m+n>-2 \text { and } m<\frac{1}{2} \text { if } r=a .
\end{aligned}
$$


The integral $G_{m, n}(r, z)$ can be put also in the following form

where

$$
G_{m, n}(r, z)=-\mathscr{I} U_{m-3, n}(r, z+i a)-a \mathscr{R} U_{m-2, n}(r, z+i a),
$$

$$
U_{m, n}(r, z)=\int_{0}^{\infty} t^{m+1} e^{-t z} J_{n}(r t) d t
$$

or (4)

$$
\begin{aligned}
& U_{m, n}(r, z)=\frac{r^{n} \Gamma(m+n+2)}{2^{n} \Gamma(n+1)\left(r^{2}+z^{2}\right)^{(m+n+2) / 2}} \times \\
& F\left(\frac{m+n+2}{2}, \frac{-m+n-1}{2} ; n+1 ; \frac{r^{2}}{r^{2}+z^{2}}\right), \\
& \quad(m+n>-2 ; z>0) .
\end{aligned}
$$

Here $F$ denotes a hypergeometric function. Formula (1.3) cannot be used for any $m$ and $n$ with $m+n>-2$ since $U_{m-3, n}(r, z)$ and $U_{m-2, n}(r, z)$ are convergent only if we have $m+n>1$ and $m+n>0$, respectively. For example, the integrals $G_{-1,0}(r, z), G_{-1,1}(r, z), G_{0,0}(r, z), G_{0,1}(r, z)$ and $G_{1,0}(r, z)$, which appear in the examples (i), (ii) and (iii) quoted above, cannot be evaluated with the aid of relation (1.3).

For the evaluation of the integrals $G_{m, n}(r, z)$ it is convenient to introduce the oblate spheroidal coordinates $\mu, \zeta$, defined by the equations

$$
r=a\left(1-\mu^{2}\right)^{\frac{1}{2}}\left(1+\zeta^{2}\right)^{\frac{1}{2}}, \quad z=a \mu \zeta .
$$

It can be seen that each pair $(r / a, z / a)$, where $0 \leqq r<\infty, 0 \leqq z<\infty$, corresponds to a unique pair $(\mu, \zeta)$ where $0 \leqq \mu \leqq 1,0 \leqq \zeta<\infty$. In terms of these coordinates we have for some expressions which appear frequently in the calculations:

$$
\begin{gathered}
z+i a=a(\mu \zeta+i), \quad\left[r^{2}+(z+i a)^{2}\right]^{\frac{1}{2}}=a(\zeta+\mu i) \\
\mathscr{I} \ln \frac{z+i a+\left[r^{2}+(z+i a)^{2}\right]^{\frac{1}{2}}}{z+\left(r^{2}+z^{2}\right)^{\frac{1}{2}}}=\operatorname{arcctg} \zeta
\end{gathered}
$$

\section{Evaluation of the integrals}

The object of the present paper is to give a method of evaluating the integrals $G_{m, n}(r, z)$, valid for any integer values of $m$ and $n$ provided that $m+n>-2$.

Putting, in (1.4), $z+i a$ instead of $z$, we obtain

$$
\int_{0}^{\infty} t^{m+1} e^{-t(z+i a)} J_{n}(r t) d t=U_{m, n}(r, z+i a), \quad(m+n>-2) .
$$

This equation, integrated with respect to $a$ from 0 to $a$, gives

$$
\int_{0}^{\infty} t^{m} e^{-t z}\left(e^{-i a t}-1\right) J_{n}(r t) d t=-i \int_{0}^{a} U_{m, n}(r, z+i \alpha) d \alpha .
$$


Multiplying both sides of this equation by $a$ and integrating once again with respect to $a$ from 0 to $a$, we obtain

$$
\begin{aligned}
\int_{0}^{\infty} t^{m-2} e^{-t z}\left(i e^{-i a t}-\frac{1}{2} i a^{2} t^{2}-i-a t e^{-i a t}\right) J_{n}(r t) d t & \\
& =\int_{0}^{a} \beta d \beta \int_{0}^{\beta} U_{m, n}(r, z+i \alpha) d \alpha .
\end{aligned}
$$

Equating real parts on both sides of this equation we get

$$
G_{m, n}(r, z)=\mathscr{R} \int_{0}^{a} \beta d \beta \int_{0}^{\beta} U_{m, n}(r, z+i \alpha) d \alpha .
$$

Putting $z+i \alpha=\lambda, i d \alpha=d \lambda$ and $z+i \beta=s, i d \beta=d s$, we get easily

$$
G_{m, n}(r, z)=\mathscr{I} \int_{z}^{z+i a}(z-s) d s \int_{z}^{s} U_{m, n}(r, \lambda) d \lambda, \quad(m+n>-2) .
$$

Thus the integrals $G_{m, n}(r, z)$ can be evaluated by carrying out the calculations in (2.2), $U_{m, n}(r, \lambda)$ being given by (1.5).

As an illustration of the application of this method we shall give here the calculations for $G_{-1,0}(r, z)$. We have from (1.5)

and thus

$$
U_{-1,0}(r, \lambda)=\left(r^{2}+\lambda^{2}\right)^{-\frac{1}{2}}
$$

$$
\int_{z}^{s} U_{-1,0}(r, \lambda) d \lambda=\ln \left[s+\left(r^{2}+s^{2}\right)^{\frac{1}{2}}\right]-\ln \left[z+\left(r^{2}+z^{2}\right)^{\frac{1}{2}}\right] .
$$

Now, taking into account that $\mathscr{I} \int_{z}^{z+i a}(z-s) d s=0$, from (2.2) we obtain

$$
G_{-1,0}(r, z)=\mathscr{I} \int_{z}^{z+i a}(z-s) \ln \left[s+\left(r^{2}+s^{2}\right)^{\frac{1}{2}}\right] d s .
$$

Integrating by parts we get

$$
G_{-1,0}(r, z)=\frac{1}{2} \mathscr{F}\left[a^{2} \ln \left\{z+i a+\left[r^{2}+(z+i a)^{2}\right]^{\frac{1}{2}}\right\}+\int_{z}^{z+i a} \frac{z^{2}-2 z s+s^{2}}{\left(r^{2}+s^{2}\right)^{\frac{1}{2}}} d s\right] .
$$

But we have

$$
\begin{gathered}
\mathscr{I} \int_{z}^{z+i a} \frac{d s}{\left(r^{2}+s^{2}\right)^{\frac{1}{2}}}=\operatorname{arcctg} \zeta, \mathscr{I} \int_{z}^{z+i a} \frac{s d s}{\left(r^{2}+s^{2}\right)^{\frac{1}{2}}}=a \mu, \\
\mathscr{I} \int_{z}^{z+i a} \frac{s^{2} d s}{\left(r^{2}+s^{2}\right)^{\frac{1}{2}}}=\frac{1}{2} a^{2}\left[\zeta\left(1+\mu^{2}\right)-\left(1-\mu^{2}\right)\left(1+\zeta^{2}\right) \operatorname{arcctg} \zeta\right],
\end{gathered}
$$

and thus, relation (2.3) becomes

$$
G_{-1,0}(r, z)=\frac{1}{4} a^{2}\left[\zeta\left(1-3 \mu^{2}\right)+\left(1-\zeta^{2}+\mu^{2}+3 \mu^{2} \zeta^{2}\right) \operatorname{arc} \operatorname{ctg} \zeta\right] .
$$

Similar calculations were carried out for several integrals of the type (1.1). The results are given in Section 4. 


\section{Recurrence relations}

The simplest recurrence relations are obtained by making use of the wellknown formula

$$
J_{n-1}(r t)+J_{n+1}(r t)=\frac{2 n}{r t} J_{n}(r t)
$$

which gives at once

$$
G_{m+1, n-1}+G_{m+1, n+1}=\frac{2 n}{r} G_{m, n}
$$

Similarly, using the result

we obtain

$$
J_{n-1}(r t)-J_{n+1}(r t)=2 J_{n}^{\prime}(r t),
$$

$$
G_{m+1, n-1}-G_{m+1, n+1}=2 \frac{\partial G_{m, n}}{\partial r} .
$$

But from (1.6) we have

$$
\frac{\partial}{\partial r}=\frac{\left(1+\zeta^{2}\right)^{\frac{1}{2}}\left(1-\mu^{2}\right)^{\frac{1}{2}}}{a\left(\mu^{2}+\zeta^{2}\right)}\left(\zeta \frac{\partial}{\partial \zeta}-\mu \frac{\partial}{\partial \mu}\right)
$$

and thus (3.2) becomes

$$
G_{m+1, n-1}-G_{m+1, n+1}=\frac{2\left(1+\zeta^{2}\right)^{\frac{1}{2}}\left(1-\mu^{2}\right)^{\frac{1}{2}}}{a\left(\mu^{2}+\zeta^{2}\right)}\left(\zeta \frac{\partial G_{m, n}}{\partial \zeta}-\mu \frac{\partial G_{m, n}}{\partial \mu}\right) .
$$

From (3.1) and (3.3) we have

$$
\begin{aligned}
& G_{m+1, n-1}=\frac{n}{r} G_{m, n}+\frac{r}{a^{2}\left(\mu^{2}+\zeta^{2}\right)}\left(\zeta \frac{\partial G_{m, n}}{\partial \zeta}-\mu \frac{\partial G_{m, n}}{\partial \mu}\right), \\
& G_{m+1, n+1}=\frac{n}{r} G_{m, n}-\frac{r}{a^{2}\left(\mu^{2}+\zeta^{2}\right)}\left(\zeta \frac{\partial G_{m, n}}{\partial \zeta}-\mu \frac{\partial G_{m, n}}{\partial \mu}\right) .
\end{aligned}
$$

Another set of recurrence relations are obtained by observing that

$$
\frac{\partial G_{m, n}}{\partial z}=-G_{m+1, n}
$$

Taking into account the fact that from (1.6) we have

$$
\frac{\partial}{\partial z}=\frac{1}{a\left(\mu^{2}+\zeta^{2}\right)}\left[\zeta\left(1-\mu^{2}\right) \frac{\partial}{\partial \mu}+\mu\left(1+\zeta^{2}\right) \frac{\zeta}{\partial \zeta}\right]
$$

relation (3.6) becomes

$$
G_{m+1, n}=-\frac{1}{a\left(\mu^{2}+\zeta^{2}\right)}\left[\zeta\left(1-\mu^{2}\right) \frac{\partial G_{m, n}}{\partial \mu}+\mu\left(1+\zeta^{2}\right) \frac{\partial G_{m, n}}{\partial \zeta}\right] \ldots \ldots
$$

4. List of some integrals of type $G_{m, n}(r, z)$

In this section we shall give the results obtained on the evaluation of some integrals of the type $G_{m, n}(r, z)$. These evaluations were carried out either with 
the method described in Section 2 or with the recurrence formulæ (3.1), (3.4), (3.5) and (3.7). Thus, we have obtained:

$$
\begin{aligned}
& G_{0,0}(r, z)=a \mu(1-\zeta \operatorname{arc} \operatorname{ctg} \zeta), \\
& G_{0,1}(r, z)=\frac{a\left(1-\mu^{2}\right)^{\frac{1}{2}}}{2\left(1+\zeta^{2}\right)^{\frac{1}{2}}}\left[\left(1+\zeta^{2}\right) \operatorname{arcctg} \zeta-\zeta\right], \\
& G_{0,2}(r, z)=\frac{a(1-\mu)(\mu+2)}{3(1+\mu)\left(1+\zeta^{2}\right)} \\
& G_{1,0}(r, z)=\operatorname{arcctg} \zeta-\frac{\zeta}{\mu^{2}+\zeta^{2}}, \\
& G_{1,1}(r, z)=\frac{\mu\left(1-\mu^{2}\right)^{\frac{1}{2}}}{\left(1+\zeta^{2}\right)^{\frac{1}{2}}\left(\mu^{2}+\zeta^{2}\right)}, \\
& G_{1,2}(r, z)=\frac{\zeta\left(1-\mu^{2}\right)}{\left(1+\zeta^{2}\right)\left(\mu^{2}+\zeta^{2}\right)}, \\
& G_{1,3}(r, z)=\frac{(1-\mu)^{\frac{3}{2}}\left[\zeta^{2}\left(3 \mu^{2}+9 \mu+8\right)-\mu(\mu+3)\right]}{3(1+\mu)^{\frac{3}{2}}\left(1+\zeta^{2}\right)^{\frac{3}{2}}\left(\mu^{2}+\zeta^{2}\right)} \text {, } \\
& G_{2,0}(r, z)=\frac{\mu\left(5 \mu^{2} \zeta^{2}-3 \zeta^{2}+\mu^{2}+\mu^{4}\right)}{a\left(\mu^{2}+\zeta^{2}\right)^{3}}, \\
& G_{2,1}(r, z)=\frac{\zeta\left(1-\mu^{2}\right)^{\frac{1}{2}}\left(5 \mu^{2} \zeta^{2}+3 \mu^{2}+\mu^{4}-\zeta^{2}\right)}{a\left(1+\zeta^{2}\right)^{\frac{1}{2}}\left(\mu^{2}+\zeta^{2}\right)^{3}}, \\
& G_{2,2}(r, z)=\frac{\mu\left(1-\mu^{2}\right)\left(5 \zeta^{4}+\mu^{2} \zeta^{2}+3 \zeta^{2}-\mu^{2}\right)}{a\left(1+\zeta^{2}\right)\left(\mu^{2}+\zeta^{2}\right)^{3}}, \\
& G_{-1,0}(r, z)=\frac{1}{4} a^{2}\left[\zeta\left(1-3 \mu^{2}\right)+\left(1-\zeta^{2}+\mu^{2}+3 \mu^{2} \zeta^{2}\right) \operatorname{arcctg} \zeta\right] \text {, } \\
& G_{-1,1}(r, z)=\frac{a^{2}(1-\mu)^{\frac{1}{2}}}{6(1+\mu)^{\frac{1}{2}}\left(1+\zeta^{2}\right)^{\frac{1}{2}}}\left[2\left(1+\mu+\mu^{2}\right)+3 \mu \zeta^{2}(1+\mu)\right. \\
& \left.-3 \mu \zeta(1+\mu)\left(1+\zeta^{2}\right) \operatorname{arcctg} \zeta\right], \\
& G_{-2,1}(r, z)=\frac{a^{3}(1-\mu)^{\frac{1}{2}}}{16(1+\mu)^{\frac{1}{2}}\left(1+\zeta^{2}\right)^{\frac{1}{2}}}\left[(1+\mu)\left(1+\zeta^{2}\right)\left(3+5 \mu^{2} \zeta^{2}+\mu^{2}-\zeta^{2}\right) \operatorname{arcctg} \zeta\right. \\
& \left.+\frac{1}{3} \zeta\left\{9-7 \mu-13 \mu^{2}-13 \mu^{3}+3 \zeta^{2}\left(1+\mu-5 \mu^{2}-5 \mu^{3}\right)\right\}\right] .
\end{aligned}
$$

\section{REFERENCES}

(1) J. W. HARding and I. N. SNeddon, The elastic stresses produced by the indentation of the plane surface of a semi-infinite elastic solid by a rigid punch, Proc. Camb. Phil. Soc., 41 (1945), 16-26.

(2) I. N. SNeddon, Fourier transforms (McGraw-Hill Book Co., New York, 1951). 
(3) G. Eason, B. Noble, I. N. SNeddon, On certain integrals of Lipschitz-Hankel type involving products of Bessel functions, Phil. Trans. Royal Soc. London, series A, 247 (1955), 529-551.

(4) G. N. Watson, The theory of Bessel functions (Cambridge University Press, 2nd edition, 1944).

INSTITUTE OF MATHEMATICS

EMINESCU 47

BUCHAREST 3, RUMANIA 